Original Article

\title{
EFFICACY OF NILOTINIB IN PATIENTS WITH CHRONIC MYELOID LEUKEMIA
}

\author{
Sajid Ali ${ }^{a}$, Tahir Mehmood ${ }^{b}$, Kausar Bano ${ }^{c}$, Muhammad Akram ${ }^{d}$, Muhammad Imran Khan ${ }^{e}$, Tehreem Fatima \\ a'Postgraduate Resident, Medical Oncology, AIMC/Jinnah Hospital, Lahore. \\ ${ }^{\mathrm{b} C}$ Consultant Medical Oncologist, FMU/Allied Hospital, Faisalabad. \\ 'Assistant Professor, Medical Oncology, AIMC/Jinnah Hospital, Lahore. \\ 'Professor and Head Department of Medical Oncology, AIMC/Jinnah Hospital, Lahore. \\ ${ }^{\mathrm{e}}$ Assistant Professor, Radiology, FJMU/Sir Ganga Ram Hospital, Lahore. \\ 'Research Assistant, Government College University, Faisalabad.
}

\section{ABSTRACT:}

OBJECTIVE: To determine the efficacy of nilotinib in patients of chronic myeloid leukemia, chronic phase, in terms of detection of BCR-ABL by FISH method.

MATERIAL AND METHODS: This study was conducted on 92 diagnosed cases of chronic myeloid leukemia at Department of Oncology, Jinnah Hospital Lahore from August 2016 to January 2017. Patients from either gender, between the ages of 20 to 60 years were included in the study while patients having diabetes and end stage renal disease with glomerular filtration rate less than 15 $\mathrm{ml} / \mathrm{min}$ were excluded. Nilotinib treatment with the standard dose (300 mg twice daily) was given to patients with chronic phase of chronic myeloid leukemia (CP-CML). Patients were monitored as recommended by the current treatment guidelines. Treatment outcome of CP-CML in terms of efficacy was assessed at the end of 6 months of treatment.

RESULT: The mean age of the patients was $38.84 \pm 11.67$ years, with male to female ratio of 1.04:1. The mean PH value of the patients was $17.05 \pm 18.53$ and efficacy was achieved in 36 $(39.13 \%)$ patients.

CONCLUSION: The efficacy of nilotinib was achieved in significant number of CML patients.

KEYWORDS: FISH, BCR-ABL, Efficacy, Nilotinib, Chronic Myeloid Leukemia.

\section{INTRODUCTION:}

Chronic myeloid leukemia (CML) defined as monoclonal myeloproliferative hematological disorder, because of the neoplastic change of the crude hematopoietic undifferentiated cells. These juvenile myeloid antecedent cells isolate and involve the bone marrow and spleen $^{[1,2]}$. CML occurs because of development and advancement in an irregular clone of cells containing a chromosome revamp known as the Philadelphia chromosome ${ }^{[2,3]}$.

In the Philadelphia chromosome, a piece of the BCR ('breakpoint cluster region') quality from chromosome is melded with the ABL ('Abelson leukemia infection') quality on chromosome ${ }^{[9]}$. The oncogenic BCR-ABL combination quality encodes the BCR-ABL combination protein. As a protein kinase it leads to myeloid cells uncontrolled proliferation ${ }^{[3,4]}$.

The introduction of Tyrosine kinase inhibitors

(TKIs) to control malignant growth has opened new horizons in this field ${ }^{[5]}$. Imatinib is used as first line treatment in perpetual stage of $\mathrm{Ph}+\mathrm{CML}(\mathrm{CP}-\mathrm{CML})$ and it has brought about high remission and survival rates ${ }^{[3,6,7]}$. Introduction of second era TKIs, particularly nilotinib, appeared to be more compelling than imatinib as first line treatment in accomplishing critical endpoints as a complete cytogenetic response (CCyR), i.e. the nonattendance of $\mathrm{Ph}+$ cells in blood and bone marrow and a Noteworthy major molecular response (MMR), i.e. the close nonappearance of BCR-ABL combination mRNA transcripts (a decrease to

Corresponding Author:

Tahir Mehmood

Consultant Medical Oncologist,

FMU/Allied Hospital, Faisalabad.

Email:drtahirbajwa82@yahoo.com 
$\leq 0.1 \%$ on the worldwide scale). By and large, a MMR is accomplished somewhere around one year sooner with the second era TKIs in contrast to imatinib ${ }^{[8,9]}$. Therapy with nilotinib at dosages of 300 or $400 \mathrm{mg} \mathrm{BD}$ brought about higher rate of CCYR and a higher rate of MMR following a year than with Imatinib at $400 \mathrm{mg}$ ( $80 \%$ versus $65 \%$ cytogenetic response rates respectively). The ideal response was BCR-ABL $<0.1 \%$ following a year of treatment. A local study conducted on imatinib in all phases of CML demonstrated CCyR and complete hematological response tantamount to western populace on imatinib in all phases of $\mathrm{CML}$ demonstrated CCyR and complete hematological response Tantamount to western populace on imatinib with major cytogenetic response $(\mathrm{Ph}<35 \%)$. The response in chronic, accelerated and blast phases were $61 \%, 57 \%$ and $28 \%$ separately and CCyRs $39.4 \%, 35.7 \%$ and $14.3 \%$ individually ${ }^{[10]}$.

\section{METHODOLOGY:}

This cross sectional study was conducted on 92 diagnosed cases of chronic myeloid leukemia at Department of Oncology Jinnah Hospital Lahore from August 2016 to January 2017. The patients aged 20-60 years and of either gender with chronic myeloid leukemia diagnosed by bone marrow criteria and nearness of BCR-
$A B L>1$ and $P h>1$ by FISH test were included. Patients having diabetes and end organ kidney disease with glomerular filtration rate of $<15$ $\mathrm{ml} / \mathrm{min}$ were excluded. Nilotinib treatment with the standard dosage ( $300 \mathrm{mg}$ twice per day) was given to patients with chronic phase of chronic myeloid leukemia (CP-CML). Patients were followed according to recent treatment guidelines. Result of treatment of Chronic Phase CML patients as far as viability was surveyed at end of a half year treatment.

\section{RESULTS:}

The mean age of the patients was $38.8 \pm 11.7$ years. There were $47(51.1 \%)$ male and 45 $(48.9 \%)$ female patients. The male to female ratio was $1.04: 1$. Finish sub-atomic reaction (CMR) was accomplished in 36 (39.1\%) patients and it was not accomplished in 56 $(60.9 \%)$ patients. The results of this study demonstrated that 17 male and 19 female patients accomplished finish sub-atomic reaction (CMR). It is Statistically significant with $\mathrm{p}$-value $=0.011$ (table-I). The results showed that 23 patients ( $\leq 40$ years of age) accomplished finish atomic reaction (CMR), likewise 13 patients ( $>40$ years of age) accomplished finish sub-atomic reaction (CMR), which is statistically insignificant with $\mathrm{p}$-value = 0.191 (table-II).

Table-I: Comparison of patients by efficacy $(n=92)$.

\begin{tabular}{|c|c|c|c|}
\hline \multirow{2}{*}{ Gender } & \multicolumn{2}{|c|}{ Efficacy } & \multirow{2}{*}{ Total } \\
\cline { 2 - 3 } & Yes & No & 47 \\
\hline Male & 17 & 30 & 45 \\
\hline Female & 19 & 26 & 92 \\
\hline Total & 36 & 56 & \\
\hline
\end{tabular}

$X^{2}=10.49 p$-value $=0.011$

Table-II: Comparison of age with efficacy $(n=92)$.

\begin{tabular}{|c|c|c|c|}
\hline \multirow{2}{*}{ Age (Years) } & \multicolumn{2}{|c|}{ Efficacy } & \multirow{2}{*}{ Total } \\
\cline { 2 - 3 } & Yes & No & \\
\hline$<40$ & 23 & 28 & 51 \\
\hline$>40$ & 13 & 28 & 41 \\
\hline Total & 46 & 56 & 92 \\
\hline
\end{tabular}

$\mathrm{X}^{2}=8.2 \mathrm{p}$-value $=0.191$ 


\section{DISCUSSION:}

The present investigation on finish atomic reaction in CML-CP through FISH following half year of Nilotinib treatment demonstrated that CMR rates are about $39.1 \%$. This study is different from other studies in respect to its duration, that is 6 months and the essential end point i.e MMR/CCYR and PCR. FISH rather than PCR was used in this study.

Another study carried out at Agha Khan Hospital was similar to this study, however, patients were pretreated with imatinib and the method of appraisal was cytogentic reaction by means of FISH as compared to FISH technique for evaluating BCR-ABL protein in this study. Still the two investigations delineated great response with nilotinib in our populace ${ }^{[11]}$.

A study conducted in China showed MMR of $52 \%$ following one year of treatment with nilotinib ${ }^{[12]}$. In another study, conducted in Turkey, 112 patients were treated with nilotinib and were evaluated for MMR following 1-year treatment. The MMR was $66.1 \%{ }^{[13]}$. The present investigation is unique because CMR was the essential end point variable, additionally study duration was 6 months. This study also delineates great response with nilotinib through MMR, however a subset investigation on this outcomes closer. If present study was extended over a period of one year with MMR as end point measure, it may have shown comparable and better results. The outcome of ENEST $1^{\text {st }}$ study in which one of the end point was MMR at the end of two years, the results showed that MMR at end of $3,12,18$ and 24 months was $29.7 \%, 56.3 \%$, $65.8 \%$ and $61.2 \%$ respectively. While $67.3 \%$ and $82.5 \%$ achieved complete cytogenetic response at end of 6 and 12 months respectively ${ }^{[14]}$. In contrast to our study, CMR was not assessed in ENEST $1^{\text {st }}$ study. Taking complete cytogentic response as surrogate for $B C R-A B L$ this investigation is supporting current CMR results which is about $40 \%$.

The GIMEMA trial ${ }^{[15]}$ demonstrated that the MMR at 3 and 12 months was $52 \%$ and $85 \%$ respectively. Combined CMR was $41 \%$, which is almost equivalent and practically identical to our study i.e. $39.1 \%$.

An investigation by Yun SM et $\mathrm{al}^{[16]}$ showed that nilotinib resembles imatinib but, as a result of its better geographical fit with BCR-ABL, it is 20 times more potent than imatinib. An examination by Cortes $\mathrm{J}$ et $\mathrm{al}^{[17]}$ portrayed that TKI treatment with imatinib, nilotinib, or dasatinib results in high response rates, huge numbers of which must be estimated at complete molecular remission.

In this study, complete molecular response (CMR) with nilotinib was accomplished in $39.1 \%$ patients of $C M L$, chronic phase, as far as location of BCR-ABL by FISH strategy. Utilization of FISH for BCR-ABL is noteworthy if PCR isn't accessible as the outcomes are in concordance with different investigations. The outcomes were comparable with past referred studies.

\section{CONCLUSION:}

Complete molecular response by FISH can be utilized as surrogate for finish complete molecular response by PCR, if patients are non affording.

\section{CONFLICT OF INTEREST:}

There is no declared conflict of interest.

\section{ETHICAL REVIEW COMMITTEE:}

Ethical review committee of the said institute has reviewed and approved this article.

\section{REFERENCES:}

1. Abruzzese E, Breccia M, Latagliata R. Second-generation tyrosine kinase inhibitors in first-line treatment of chronic myeloid leukaemia (CML). Bio Drugs Clin immunother Biopharmac Gene Therap 2014; 28(1):17-26.

2. Brahmbhatt MM, Trivedi PJ, Patel DM, Shukla SN, Patel PS. Location of the BCR/ ABL Fusion Genes on Both Chromosomes 9 in $\mathrm{Ph}$ negative young CML Patients: An Indian Experience. Indian $\mathrm{J}$ Hematolo Blood transfus 2014; 30(4):241-6.

3. Nair V, Sharma A, Kotwal J, Bhikshapathy M, Mishra DK, Das S, et al. Monitoring of response to therapy with imatinib mesylate in Chronic Myeloid Leukemia in chronic phase (CML-CP). Med J Armed Forces India 
$2014 ; 70(4): 315-20$.

4. Takahashi N, Miura M, Kuroki J, Mitani K, Kitabayashi A, Sasaki O, et al. Multicenter phase II clinical trial of nilotinib for patients with imatinib-resistant or -intolerant chronic myeloid leukemia from the East Japan CML study group evaluation of molecular response and the efficacy and safety of nilotinib. Biomarker Res 2014;2 $(1): 6$.

5. Boons CC, Swart EL, Timmers L, van de Ven PM, Janssen JJ, Hugtenburg JG. Study protocol of the RAND-study: a multicenter, prospective cohort study investigating response and adherence to nilotinib treatment in chronic myeloid leukemia. BMC Cancer 2014;14:247.

6. Ostendorf BN, le Coutre P, Kim TD, QuintasCardama A. Nilotinib recent results in cancer research. Fortschritte der Krebsforschung Progress dans les recherches sur le cancer 2014;201:67-80.

7. Goldman JM, Marin D. Is imatinib still an acceptable first-line treatment for CML in chronic phase? Oncology (Williston Park, NY). 2012;26(10):901-7.

8. Cortes J, Hochhaus A, Hughes T, Kantarjian $\mathrm{H}$. Front-line and salvage therapies with tyrosine kinase inhibitors and other treatments in chronic myeloid leukemia. J Clin Oncol2011;29(5):524-31.

9. Jabbour E, Kantarjian HM, O'Brien S, Shan J, Quintas-Cardama A, Garcia-Manero G, et al. Front-line therapy with secondgeneration tyrosine kinase inhibitors in patients with early chronic phase chronic myeloid leukemia: what is the optimal response? J Clin Oncol 2011;29(32):42605.

10. Aziz Z, Treatment of chronic myeloid leukemia in the imatinib era perspective from a developing country 2018.

11. Hussain S, Shaikh UM. Response and adverse effects of Nilotinib in Imatinibresistant chronic myeloid leukemia patients: Data from a developing country. Clin Therap 2015; 37(11):2449-57.

12. Wang J, Shen ZX, Saglio G, Jin J, Huang $H$, $\mathrm{Hu} Y$, et al. Phase 3 study of nilotinib vs imatinib in Chinese patients with newly diagnosed chronic myeloid leukemia in chronic phase: ENEST China. Blood.
2015;125(18):2771-8.

13. Saydam G, Haznedaroglu IC, Kaynar L, Yavuz AS, Ali R, Guvenc B, et al. Outcomes with frontlinenilotinibtreatment in Turkish patients with newly diagnosed Philadelphia chromosome-positive chronic myeloid leukemia in chronic phase.2016;17 (14):1851-8.

14. Hochhaus AGJ. Frontline nilotinib in patients with chronic myeloid leukemia in chronic phase: results from the European ENEST1st study. Leukemia 2016; 30(1): 57-64.

15. Rosti GFC. Excellent outcomes at 3 years with Nilotinib $800 \mathrm{mg}$ daily in early chronic phase, $\mathrm{Ph}+$ Chronic Myeloid Leukemia (CML): Results of a Phase 2 GIMEMA CML WP Clinical Trial. Blood 2010; 116:359.

16. Yun SM, Jung KH, Kim SJ, Fang Z, Son MK, Yan $\mathrm{HH}$, et al. HS-438, a new inhibitor of imatinib-resistant BCR-ABL T315I mutation in chronic myeloid leukemia. Cancer Lett. 2014 Jun 28;348(1-2):50-60.

17. Cortes J, QuintasCardama A, Kantarjian HM. Monitoring molecular response in chronic myeloid leukemia. Cancer 2011; 117(6):1113-22.

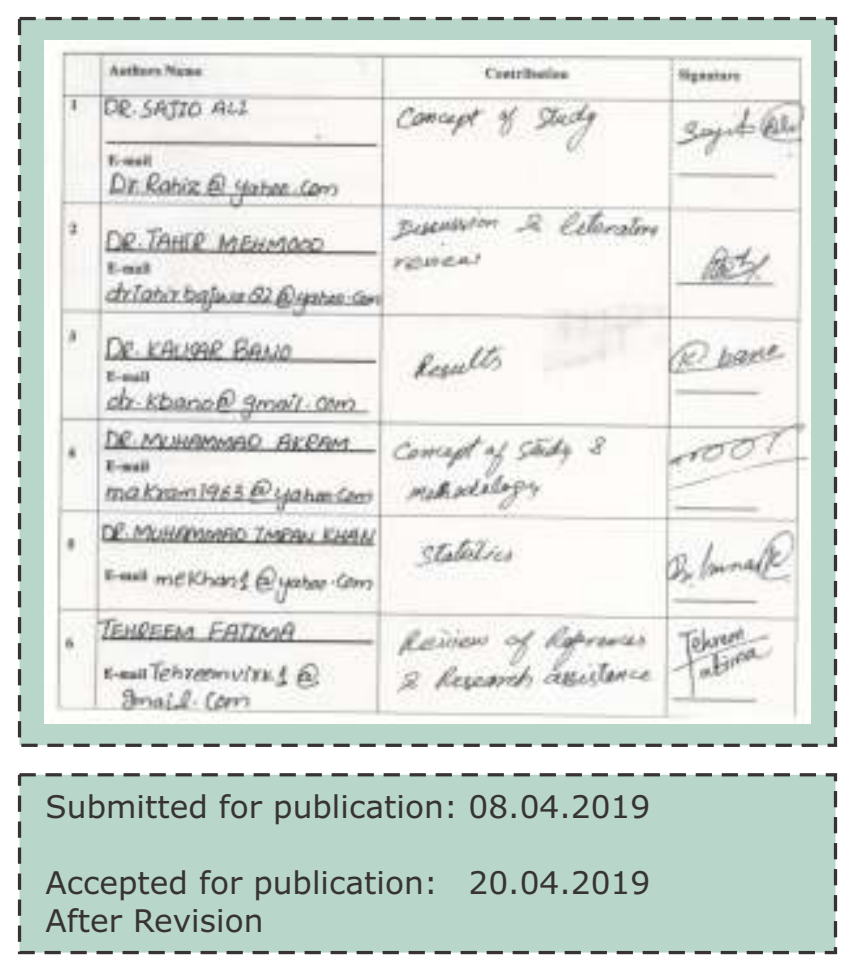

